\title{
Paramagnon-induced dispersion anomalies in the cuprates
}

\author{
R.S. Markiewicz, S. Sahrakorpi, and A. Bansil \\ Physics Department, Northeastern University, Boston MA 02115
}

(Dated: July 13, 2018)

\begin{abstract}
We report the self-energy associated with RPA magnetic susceptibility in the hole-doped $\mathrm{Bi}_{2} \mathrm{Sr}_{2} \mathrm{CuO}_{6}(\mathrm{Bi} 2201)$ and the electron-doped $\mathrm{Nd}_{2-x} \mathrm{Ce}_{x} \mathrm{CuO}_{4}(\mathrm{NCCO})$ in the overdoped regime within the framework of a one-band Hubbard model. Strong weight is found in the magnetic spectrum around $(\pi, 0)$ at about $360 \mathrm{meV}$ in Bi2201 and $640 \mathrm{meV}$ in NCCO, which yields dispersion anomalies in accord with the recently observed 'waterfall' effects in the cuprates.
\end{abstract}

PACS numbers: 79.60.-i, 71.38.Cn, 74.72.-h, 71.45.Gm

Very recent angle-resolved photoemission (ARPES) experiments in the cuprates have revealed the presence of an intermediate energy scale in the $300-800 \mathrm{meV}$ range where spectral peaks disperse and broaden rapidly with momentum, giving this anomalous dispersion the appearance of a 'waterfall' 1, 2, 3, 4, 5, 6]. Similar self-energies have also been adduced from optical data 7]. This new energy scale is to be contrasted with the well-known low energy 'kinks' in the 50-70 meV range, which have been discussed frequently in the cuprates as arising from the bosonic coupling of the electronic system with either phonons [8] and/or magnetic modes [9]. Although low energy plasmons [10, 11] are an obvious choice for the new boson, analysis indicates that the plasmons lie at too high an energy of $\sim 1 \mathrm{eV}$ to constitute a viable candidate 12 . Here we demonstrate that paramagnons provide not only an explanation of the energy scale but also of the other observed characteristics of the waterfall effect in both hole and electron doped cuprates.

For this purpose, we have evaluated the self-energy associated with the RPA magnetic susceptibility in the hole-doped $\mathrm{Bi}_{2} \mathrm{Sr}_{2} \mathrm{CuO}_{6}$ (Bi2201) and the electron-doped $\mathrm{Nd}_{2-x} \mathrm{Ce}_{x} \mathrm{CuO}_{4}$ (NCCO). [13] In order to keep the computations manageable, the treatment is restricted to the overdoped systems where magnetic instabilities are not expected to present a complication. Our analysis proceeds within the framework of the one-band Hubbard Hamiltonian, where the bare band is fit to the tightbinding LDA dispersion [14, 15]. We incorporate selfconsistency by calculating the self energy and susceptibility using an approximate renormalized one-particle Green function

$$
G=\bar{Z} /\left(\omega-\bar{\xi}_{k}+i \delta\right),
$$

where $\bar{\xi}_{k}=\bar{Z}\left(\epsilon_{k}-\mu\right)$. Here, $\epsilon_{k}$ are bare energies and $\mu$ is the chemical potential, and the renormalization factor is $\bar{Z} \sim\left(1-\partial \Sigma^{\prime} / \partial \omega\right)^{-1}<1$. The associated magnetic susceptibility is

$$
\chi_{0}(\vec{q}, \omega)=-\bar{Z}^{2} \sum_{\vec{k}} \frac{\bar{f}_{\vec{k}}-\bar{f}_{\vec{k}+\vec{q}}}{\bar{\epsilon}_{\vec{k}}-\bar{\epsilon}_{\vec{k}+\vec{q}}+\omega+i \delta},
$$

where $\delta$ is a positive infinitesimal, $\bar{f}_{\vec{k}} \equiv f\left(\bar{\epsilon}_{\vec{k}}\right)$ is the Fermi function. The RPA susceptibility is given by

$$
\chi(\vec{q}, \omega)=\frac{\chi_{0}(\vec{q}, \omega)}{1-U \chi_{0}(\vec{q}, \omega)},
$$

with $U$ denoting the Hubbard parameter. The self-energy can be obtained straightforwardly from the susceptibility via the expression [16] (at $T=0)$

$$
\begin{array}{r}
\Sigma(\vec{k}, \omega)=\frac{3}{2} \bar{Z} U^{2} \sum_{\vec{q}} \int_{0}^{\infty} \frac{d \omega^{\prime}}{\pi} \operatorname{Im} \chi\left(\vec{q}, \omega^{\prime}\right) \\
\quad \times\left[\frac{\bar{f}_{\vec{k}-\vec{q}}}{\omega-\bar{\xi}_{\vec{k}-\vec{q}}+\omega^{\prime}}+\frac{1-\bar{f}_{\vec{k}-\vec{q}}}{\omega-\bar{\xi}_{\vec{k}-\vec{q}}-\omega^{\prime}}\right] .
\end{array}
$$

Concerning technical details, we note that for the generic purposes of this study, all computations in this article employ a fixed value $\bar{Z}=0.5$, which is representative of the band dispersions observed experimentally in hole as well as electron doped cuprates. 17. Self-consistency is then achieved approximately by determining values of the chemical potential $\mu$ and the Hubbard parameter $U$ to keep a fixed doping level and to ensure that the bands are indeed renormalized by the average factor $\bar{Z}=0.5$. The procedure is relatively simple, but it should capture the essential physics of the electron-paramagnon interaction, although our treatment neglects the energy [18] and momentum dependencies of $\bar{Z}$. Note also that in the overdoped regime considered, the effective $U$ values in Bi2201 and NCCO are small enough that the system remains paramagnetic and the complications of the antiferromagnetic instability are circumvented. Specifically, the presented results on $\mathrm{Bi} 2201$ are for $x=0.27$ with $\mu=-0.43 \mathrm{eV}$ and $U=3.2 t$, while for NCCO, $x=-0.25$ with $\mu=0.18 \mathrm{eV}$ and $U=4 t$.

Figure 1 summarizes the results for Bi2201. We consider Figs. 1(a) and (b) first, which give the real and imaginary parts of the self-energy at several different momenta as a function of frequency. The theoretical self-energies, which refer to Bi2201, should be compared directly with the corresponding experimental data (gold squares [3]), although available experimental points for $\mathrm{Bi} 2212$ [4] and $\mathrm{La}_{2-x} \mathrm{Sr}_{x} \mathrm{CuO}_{4}$ (LSCO) [5] are also included for completeness. The agreement between theory 
and experiment is seen to be quite good for the real part of the self-energy in (a), while theory underestimates the imaginary part of the self-energy by a factor of $\sim 2$. That the computed $\Sigma^{\prime \prime}$ is smaller than the experimental one is to be generally expected since our calculations do not account for scattering effects beyond those of the paramagnons. Here, we should keep in mind that there are uncertainties inherent in the experimental self-energies due to different assumptions invoked by various authors concerning the bare dispersions in analyzing the data. In particular, Feng et al. [4] extract the bare dispersion by assuming that $\Sigma^{\prime}$ is always positive and goes to zero at large energies. Other groups [3, 19] compare their results to LDA calculations and argue that $\Sigma^{\prime}$ must become negative at higher energies. Our computed $\Sigma^{\prime}$ in Fig. 1(a) becomes negative over the range $0.35-0.9 \mathrm{eV}$ in certain $\vec{k}$ directions. Interestingly, various computed colored lines in (a) and (b) more or less fall on top of one another, indicating that the self-energy is relatively insensitive to momentum, especially below the Fermi level, consistent with experimental findings [5], even though $\Sigma$ possesses a fairly strong frequency dependence.

Fig. 1(c) gives further insight into the nature of the spectral intensity obtained from the self energy of Eq. 4. The spectral intensity shown in the color plot of the figure is representative of the ARPES spectrum, matrix element effects 20] notwithstanding. The peak of the spectral density function defined by taking momentum density cuts (MDCs), shown by yellow dots, follows the renormalized dispersion (orange dashed line) up to binding energy of about $200 \mathrm{meV}$. It then disperses to higher energies rapidly (waterfall effect) as it catches up with the bare dispersion (red solid line) around $\Gamma$. In fact, near $\Gamma$, the dressed spectral peak lies slightly below the bare band. The width of the spectral function is largest in the intermediate energy range of $200-600 \mathrm{meV}$, where its slope also is the largest. This behavior of the spectral function results from the presence of peaks in the real and imaginary parts of the self-energy in the 200$500 \mathrm{meV}$ energy range discussed in connections with Figs. 1 (a) and (b) above. It is also in accord with the waterfall effect observed in ARPES experiments, although the sharpness of the theoretically predicted waterfall in Fig. 1 (c) is less severe than in experiments, which may be due to limitations of our model, including the approximations underlying our treatment of the susceptibility.

Fig. 2 considers the case of electron doped (overdoped) NCCO. The peak in $\Sigma^{\prime}$ in Fig. 2(a) lies at binding energies of $0.5-0.6 \mathrm{eV}$ (in different $\vec{k}$-directions) with a height of $0.55-0.7 \mathrm{eV}$. Correspondingly, the peak in $\Sigma^{\prime \prime}$ in Fig. 2(b) lies at a binding energy of $0.7-1.1 \mathrm{eV}$ with a height of 1-1.4 eV. Comparing these with the results of Fig. 1, we see that the self-energy effects in NCCO are much larger than in Bi2201. Our computed shift of $\sim 300 \mathrm{meV}$ in the position of the peak in $\Sigma^{\prime}$ to higher binding en-
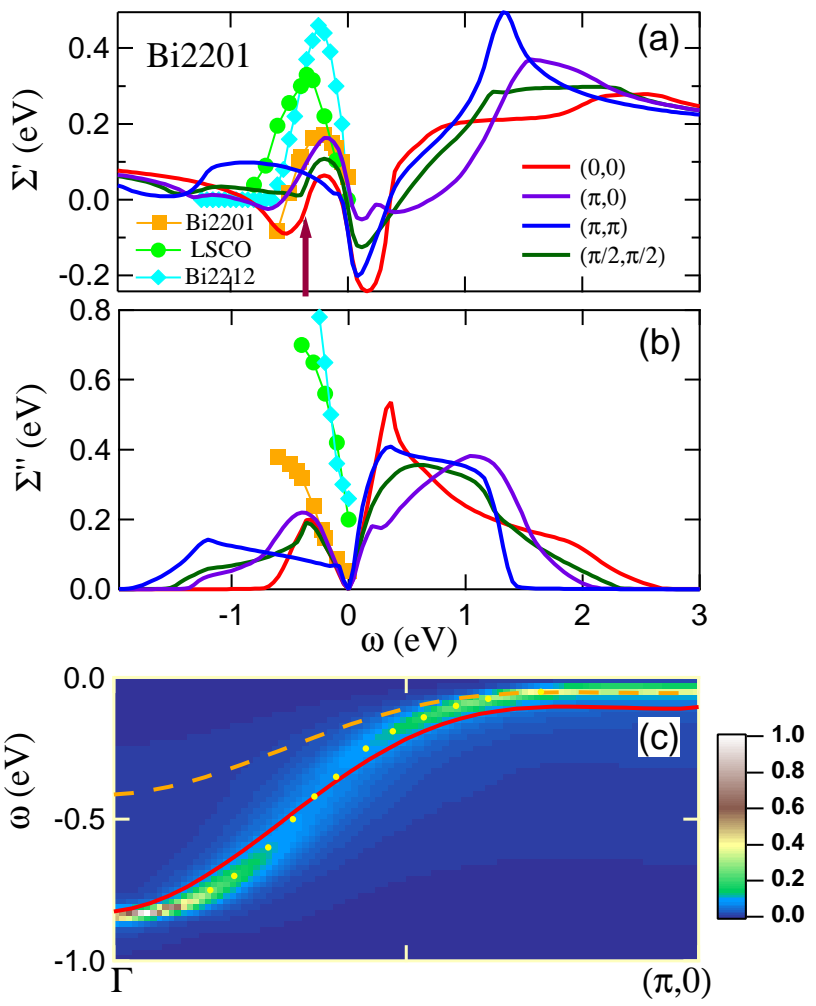

FIG. 1: (Color online) (a) and (b): Real and imaginary parts $\Sigma^{\prime}$ and $\Sigma^{\prime \prime}$, respectively, of the paramagnon self-energy in overdoped Bi2201. Theoretical results at various momenta are shown by lines of different colors. The values of $\Sigma^{\prime}$ have been shifted by a constant, to produce a zero average value of the theoretical $\Sigma^{\prime}$ at the Fermi level. Experimental points are from the nodal point for: Bi2201 (gold squares, Ref. [3); LSCO (circles, Ref. 5); and Bi2212 (diamonds, Ref. 4). Thin lines joining experimental points are guides to the eye. Arrow marks the location of peak in $\chi^{\prime \prime}$ at $(\pi, 0)$. (c): Spectral density in the energy-momentum plane obtained from the dressed Green function is shown in a color plot along with the bare (red line) and the renormalized (dashed orange line) dispersions. Dots mark the peak positions of the MDC plots of the dressed spectral density.

ergy in going from Bi2201 to NCCO is in good accord with the experimentally reported shift of $\sim 300 \mathrm{meV}[\underline{6}]$. The dispersion underlying the dressed Green function, which may be tracked through the yellow dots, is highly anomalous and presents a kink-like feature quite reminiscent of the more familiar low energy kinks in the $50 \mathrm{meV}$ range around the $(\pi, 0)$-direction 21], which have been discussed frequently in the cuprates. This strong bosonic coupling is also reflected in the fact that the band bottom in NCCO lies several hundred meVs below the bare LDA band in Fig. 2(c). It is interesting to note that the self-energies of Figs. 1 and 2 display a 'mirror-like' symmetry: The peaks below the Fermi energy in $\Sigma^{\prime}$ and $\Sigma^{\prime \prime}$ for Bi2201 in Fig. 1 are smaller than those above the Fermi energy, but the situation reverses itself for NCCO 

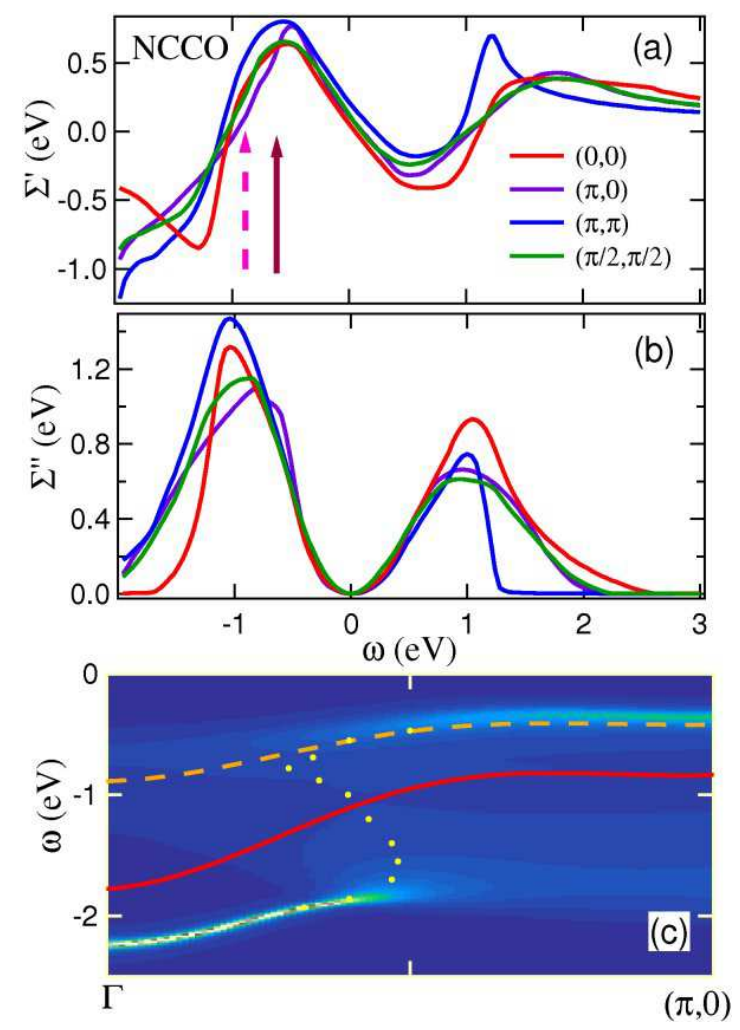

FIG. 2: (Color online) Same as the caption to Fig. 1, except that this figure shows only the computed results for overdoped NCCO. Experimental self-energies are not available and are therefore not shown (see text). Solid (dashed) arrow in (a) shows location of peak in $\chi^{\prime \prime}$ at $(\pi, 0)((\pi / 2, \pi / 2))$.

in Fig. 2 in that now the peaks below the Fermi energy become larger than those above the Fermi energy.

The aforementioned shift of the peak in $\Sigma^{\prime}$ to higher energy in NCCO can be understood in terms of the characteristics of the magnetic susceptibility. Figure 3 compares in Bi2201 and NCCO the imaginary part $\chi^{\prime \prime}$, which is seen from Eq. 4 to be related directly to the real as well as imaginary part of the self-energy. $\chi^{\prime \prime}$ is seen to be quite similar in shape along the $\Gamma$ to $(\pi, 0)$ line in Bi2201 and NCCO, except that in NCCO the band of high intensity (the yellowish trace) extends to a significantly higher energy scale. In contrast, $\chi^{\prime \prime}$ in the two systems differs sharply around $(\pi, \pi)$. These differences reflect those in the low-energy magnetic response of the two cuprates. NCCO with strong magnetic response around $(\pi, \pi)$ exhibits a nearly commensurate AFM order, while Bi2201 is very incommensurate, with peaks shifted toward $(\pi, 0)$. In fact, the high energy peaks in the selfenergy in Figs. 1 and 2 are tied to the flat-tops near $(\pi, 0)$ at $\omega_{1} \sim 0.36 \mathrm{eV}$ in Bi2201 (solid arrow), and near both $(\pi, 0)$ at $\omega_{2}=0.62 \mathrm{eV}$ (solid arrow) and $(\pi / 2, \pi / 2)$ at $\omega_{3}=0.9 \mathrm{eV}$ (dashed arrow) in NCCO. Above these energies the weight in $\chi^{\prime \prime}$ falls rapidly, going to zero near

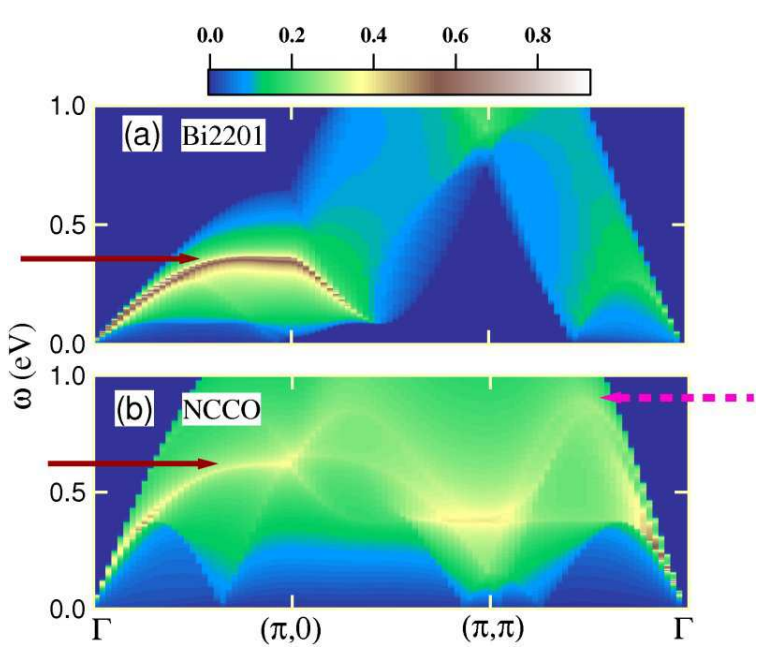

FIG. 3: (Color online) Map of the imaginary part of the magnetic susceptibility for (a) hole-doped Bi2201 and (b) electrondoped NCCO. Spectral weights are in units of $e V^{-1}$. Arrows mark the positions of the high spectral weights discussed in the text.

an energy $8 \bar{t}$.

A reference to Figs. 1(a) and 2(a), where the energy $\omega_{1}$ in Bi2201, and the energies $\omega_{2}$ and $\omega_{3}$ in NCCO are marked by arrows, indicates that the peaks in $\Sigma^{\prime}$ are correlated with these features in the magnetic susceptibility. In this spirit, the shift in the peak in $\Sigma^{\prime}$ to higher energy in going from Bi2201 to NCCO reflects the fact that feature $\omega_{3}$ in $\chi^{\prime \prime}$ at $(\pi / 2, \pi / 2)$ in NCCO (dashed arrow in Fig. 2(a)) lies at a higher energy than the $(\pi, 0)$ feature $\omega_{1}$ in Bi2201 (arrow in Fig. 1(a)). Notably, when the Stoner factor $S=1 /\left(1-U \chi_{0}\right)$ is large, a peak in $\chi^{\prime \prime}$ arises from a peak in $\chi_{0}^{\prime}(\omega)$, which in turn is associated with nesting of features separated by $\omega$ in energy. In the present case, the nesting is from unoccupied states near the Van Hove singularity (VHS) at $(\pi, 0)$ to the vicinity of the band bottom at $\Gamma$, so $\omega_{1} \sim 2\left(t+2 t^{\prime}\right) \sim 0.32 \mathrm{eV}$ in Bi2201. The larger value of $\omega_{2}$ in NCCO reflects the shift of the Fermi energy to higher energies in an electron-doped material.

A notable difference between electron and hole doping is the low- $\omega$ behavior of $\Sigma^{\prime \prime}$, which is quadratic in $\omega$ for electron-doping in Fig. 2(b), but nearly linear for hole-doping in Fig. 1(b). The linearity for hole-doping, reminiscent of marginal Fermi liquid physics, is associated here with the proximity of the chemical potential to the VHS. This point is considered further in Fig. 4 where $\Sigma^{\prime \prime}$ is shown in Bi2201 at the $(\pi, 0)$ point for three different values of the chemical potential. When the chemical potential lies at the VHS (red line), $\Sigma^{\prime \prime}$ varies linearly, but when it is shifted by $75 \mathrm{meV}$ above or below the VHS, the behavior changes rapidly to become parabolic.

The strong magnetic scattering discussed in this study in the case of overdoped cuprates should persist into the 


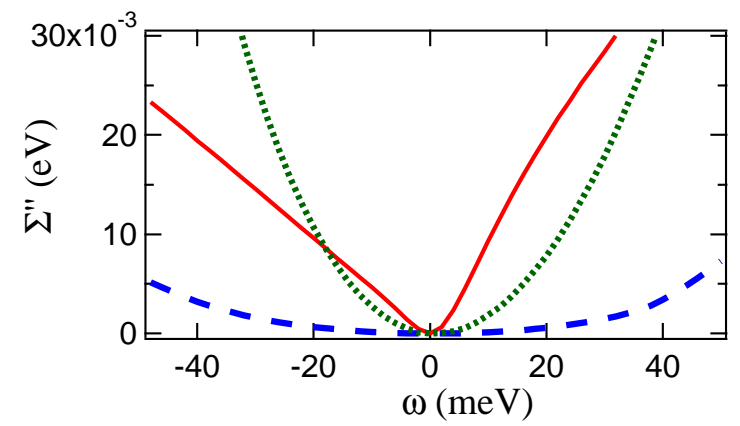

FIG. 4: (Color online) Low-energy behavior of $\Sigma^{\prime \prime}(\omega)$ at $(\pi, 0)$ in Bi2201 for three different values of the chemical potential $\mu$ in relation to the position of the energy $E_{V H S}$ of the van Hove singularity. $E_{V H S}-\mu=0$ (red solid line); $E_{V H S}-\mu=$ $+75 \mathrm{meV}$ (green dotted line); and $E_{V H S}-\mu=-75 \mathrm{meV}$ (blue dashed line).

underdoped regime, where the Stoner factor is expected to become larger. In fact, this scattering is a precursor to the magnetically ordered state near half-filling and it is responsible for opening the magnetic gap. In contrast, a number of authors have related the presence of waterfall-like effects near half-filling to 'Mott' physics associated with $(\pi, \pi)$ AFM fluctuations 22, 23, 24], but have difficulty explaining why these effects persist into the overdoped regime.

The possible doping dependence of $U$ has been an important issue in connection with electron-doped cuprates. A doping-dependent $U$ is suggested by a number of studies in the hole-doped cuprates as well. These include: Optical evidence of Mott gap decrease 25]; ARPES observation of very LDA-like bands in optimally and overdoped materials; models of the magnetic resonance peak 26]; and, a strongly doping-dependent gap derived from Hall effect studies [27]. The $\bar{Z}^{2}$-renormalization of $\chi_{0}$ in Eq. 2 bears on this question and gives insight into how the value of $U$ enters into the magnetic response of the system. Recall that the susceptibility is often evaluated in the literature via Eq. 3 using experimental band parameters, but without the $\bar{Z}^{2}$ factor of Eq. 2 in $\chi_{0}$, which yields a $\chi$ scaling $\sim \bar{Z}^{-1}$ rather than the correct scaling of $\chi \sim \bar{Z}$. This can be corrected by replacing the $U$ in the Stoner factor by

$$
U_{e f f}=\bar{Z}^{2} U
$$

Indeed, our Hubbard parameter for $\mathrm{NCCO}$ of $U=4 t$ is closer to the value at half-filling than is generally found. [28]

In conclusion, we have shown that the higher energy magnetic susceptibility in the cuprates has considerable weight near $(\pi, 0)$ and that this leads to a high energy kink or waterfall-like effect in dispersion in both elec- tron and hole-doped cuprates, providing an explanation of such effects observed recently in ARPES. Although our analysis is limited to the overdoped regime, we expect strong magnetic scattering to persist into the underdoped regime. This point however bears further study.

This work is supported by the US Department of Energy contract DE-AC03-76SF00098 and benefited from the allocation of supercomputer time at NERSC and Northeastern University's Advanced Scientific Computation Center (ASCC).

[1] F. Ronning et al., Phys. Rev. B71, 094518 (2005).

[2] J. Graf et al., to be published, Phys. Rev. Lett.

[3] W. Meevasana et al., unpublished.

[4] B.P. Xie, et al., cond-mat/0607450.

[5] T. Valla et al., cond-mat/0610271.

[6] Z.-H. Panet al., cond-mat/0610442.

[7] J. Hwang et al., cond-mat/0610488.

[8] A. Lanzara et al., Nature (London) 412, 510 (2001); X.J. Zhou, et al., Phys. Rev. Lett. 95, 117001 (2005).

[9] A. Kaminski et al., Phys. Rev. Lett. 86, 1070 (2001); P.D. Johnson et al., Phys. Rev. Lett. 87, 177007 (2001); S.V. Borisenko et al., Phys. Rev. Lett. 90, 207001 (2003); A.D. Gromko et al., Phys. Rev. B68, 174520 (2003).

[10] N. Nücker et al., Phys. Rev. B39, 12379 (1989).

[11] Y.Y. Wang et al., Phys. Rev. Lett. 77, 1809 (1996).

[12] R.S. Markiewicz and A. Bansil, to be published, Phys. Rev. B.

[13] Although not discussed here, we find similar results in LSCO.

[14] R.S. Markiewicz et al., Phys. Rev. B72, 054519 (2005).

[15] For Bi2201, we use the parameters of $\mathrm{Bi}_{2} \mathrm{Sr}_{2} \mathrm{CaCu}_{2} \mathrm{O}_{8}$ (Bi2212), but neglect the bilayer splitting. Following Ref. 14, the hopping parameters are $\left(t, t^{\prime}, t^{\prime \prime}, t^{\prime \prime \prime}\right)=(360,-$ $100,35,10) \mathrm{meV}$ for Bi2201 and $(420,-100,65,7.5) \mathrm{meV}$ for NCCO.

[16] W.F. Brinkman and S. Engelsberg, Phys. Rev. 169, 417 (1968).

[17] The specific values of $Z$ are 0.28 in $\operatorname{Bi2212}$ and 0.55 in NCCO. 14]

[18] Specifically, $\bar{Z}$ is the renormalization of the coherent part of the dispersion.

[19] A. Lanzara, personal communication.

[20] S. Sahrakorpi et al., Phys. Rev. Lett. 95, 157601 (2005).

[21] A. Kaminski et al., Phys. Rev. Lett. 86, 1070 (2001).

[22] Y. Kakehashi and P. Fulde, J. Phys. Soc. Japan 74, 2397 (2005).

[23] E. Manousakis, cond-mat/0608467

[24] Q.-H. Wang, F. Tan, and Y. Wan, cond-mat/0610491.

[25] S. Uchida et al., Phys. Rev. B43, 7942 (1991).

[26] H. Woo et al., Nature Physics, 2, 600 (2006), in supplementary materials.

[27] S. Ono, S. Komiya, and Y. Ando, cond-mat/0610361 The stronger doping dependence may be due to the high measurement temperatures.

[28] The smaller $U$ for Bi2201 may be due to neglect of $k_{z^{-}}$ dispersion, which broadens features in $\chi$ near the VHS. 